\title{
World-smallest fiber-GRIN lens system for optofluidic applications
}

\author{
Adam Filipkowski, ${ }^{1}$ Bernard Piechal, ${ }^{1}$ Dariusz Pysz, ${ }^{1}$ Ryszard Stepien, ${ }^{1}$ Jarosław Cimek ${ }^{1,2}$, Andrew Waddie, ${ }^{3}$ \\ Mariusz Klimczak, ${ }^{1}$ Paulina Stafiej, ${ }^{4}$ Mohammad R. Taghizadeh, ${ }^{3}$ and Ryszard Buczynski. ${ }^{1,2,3^{*}}$ \\ ${ }^{1}$ Department of Glass, Institute of Electronic Materials Technology, Wolczynska 133, 01-919 Warsaw, Poland \\ ${ }^{2}$ Faculty of Physics, University of Warsaw, Pasteura 7, 02-093, Warsaw, Poland \\ ${ }^{3}$ Department of Physics, School of Engineering and Physical Sciences, Heriot-Watt University, Scottish Universities \\ Physics Alliance, Edinburgh, EH14 4AS, UK \\ ${ }^{4}$ Faculty of Physics, Nicolaus Copernicus University, Grudziadzka 5, 87-10 Torun, Poland
}

Received May 30, 2016; accepted June 28, 2016; published June 30, 2016

\begin{abstract}
We have developed a new type of optical fiber probe which integrates a standard single mode fiber with a gradient index (GRIN) microlens. The system is perfectly suited for optofluidic sensor applications since the diameter of the lens module is exactly the same as that of the optical fiber. Moreover, the performance of the GRIN lens is not degraded by low contrast of the refractive index between the lens and the fluidic environment. The GRIN lens is made with a novel technology of nanostructured optics.
\end{abstract}

Recently rapid growth of interest has been observed in the development and practical applications of optofluidic systems [1]. The integration of a microlens with an optical fiber allows to miniaturize optofluidical systems relying on air lenses for light focusing from optical fibers [2] which offer relatively low optical power and hence require a significant distance from focus points. Various methods have been developed for the termination of optical fibers with microlenses. These include precision ion milling of glass with a focused ion beam, direct 3D printing with a laser beam focused in UV-curable epoxy, or two-step etching of fiber in hydrogen fluoride (HF) with an intermediate step of polishing using an $\mathrm{Ag}+$ ion beam. These methods offer classical, refractive type microlenses, the optical properties of which are dependent on the surrounding optical medium and hence of limited performance when immersed in liquids.

An alternative solution is to use gradient index (GRIN) optical components where focusing takes place in the bulk of such an element and not on its surface. GRIN lenses have optical properties much less dependent on the refractive index of an external medium, which is advantageous in optofluidic applications. Classical methods of GRIN development cannot meet the requirements of high gradients with the parabolic refractive index distribution required for microlenses.

A concept of nanostructured GRIN microoptics can be used for this purpose [3-4]. The operation principle of nanostructured elements can be described by the

\footnotetext{
*E-mail: ryszard.buczynski@itme.edu.pl.
}

effective medium theory (EMT) [5]. The basic MaxwellGarnet EMT, which gives the effective permittivity of a composite structure composed of two materials $\left(\varepsilon_{\mathrm{m}}\right.$ and $\varepsilon_{\mathrm{i}}$ with relative proportions of $(1-\delta)$ and $\delta$ respectively), is expressed numerically as

$$
\varepsilon_{e}=\varepsilon_{m} \frac{\varepsilon_{i}(1+2 \delta)-\varepsilon_{m}(2 \delta-2)}{\varepsilon_{m}(2-\delta)+\varepsilon_{i}(1-\delta)},
$$

where $\varepsilon_{\mathrm{i}}$ and $\varepsilon_{\mathrm{m}}$ are the relative permitivities of the inclusion and matrix materials respectively and $\delta$ is the proportion of an inclusion material in the composite structure. This first order theory provides sufficient accuracy for the design of a microlens.

The nanostructured GRIN lens is constructed of discrete sub-wavelength sized glass rods made of two different glasses, arranged in a certain pattern that mimics continuous gradient index distribution. The optical properties of the resulting lens depend on that pattern, on the refractive indices of two types of rods and on their size. In our structures, individual rods are sized below $1 / 5^{\text {th }}$ of the wavelength of the propagating light. According to EMT, they are not experienced by the propagating light as individual discrete rods, but as an effective averaged medium with refractive index distribution defined by high refractive index rods distribution among low refractive index rods (Fig. 1).
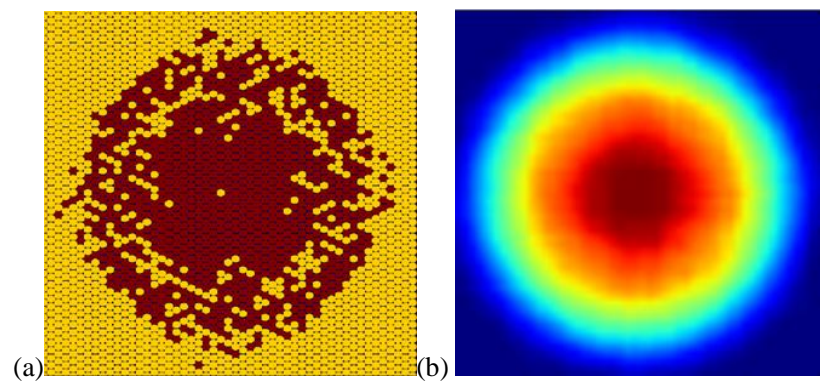

Fig. 1. Concept of nanostructured GRIN lens: (a) discrete structure of lens composed of two types of subwavelength rods, (b) effective refractive index distribution in a nanostructured GRIN lens. 
The Fast Fourier transform Beam Propagation Method (FFT BPM) was used to simulate different variations of the optical element attached to the fiber. A set of simulations was performed for an nGRIN lens attached to the fiber. The fiber that we use in those simulations has a core diameter of $4.6 \mu \mathrm{m}$ and $400 \mu \mathrm{m}$ length. The refractive index of the core is $n_{\text {core }}=1.452$. The cladding has a refractive index $n_{\text {cladding }}=1.446$. In addition, a solid glass spacer is placed between the fibre and the nanostructured GRIN lens. The spacer enables aperture matching between the fiber core and the aperture of the lens. The refractive index of the spacer glass is similar to the low refractive index glass in the nanostructured GRIN lens. We have optimised the length of the spacer and the lens to obtain sharp focusing in the free space behind the lens. As a light source we use a $1550 \mathrm{~nm}$ Gaussian beam, which is $4.6 \mu \mathrm{m}$ wide. The glasses used in the structure of the lens have refractive indices of $n_{l}=1.55$ and $n_{2}=1.5101$.

(a)

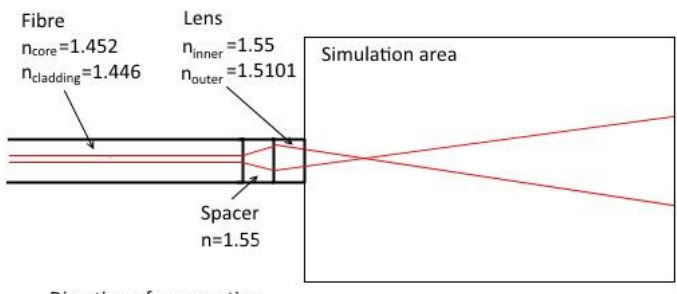

Direction of propagation

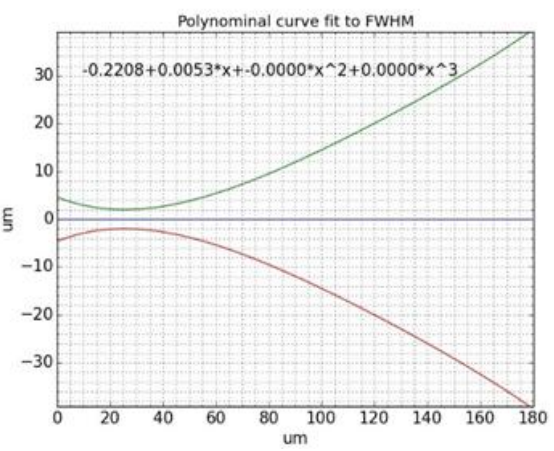

(b)

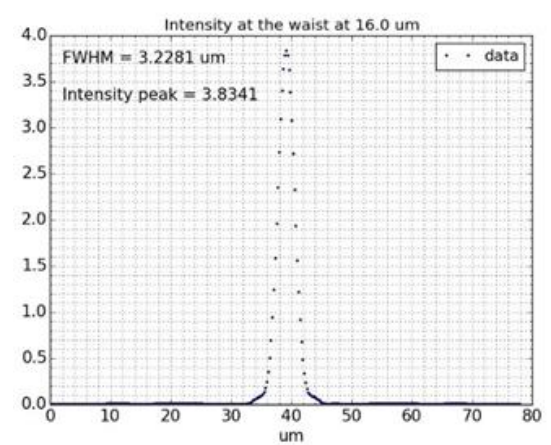

(c)

Fig. 2. Beam propagation after end plane of the nanostructured lens attached to the fiber with a spacer positioned in-between. The fiber has a core diameter of $4.6 \mu \mathrm{m}$ and length of $400 \mu \mathrm{m}$. (a) Schematic of the simulation setup; (b) evolution of beam diameter behind the nanostructured lens, (c) Intensity distribution at the beam waist.
The FFT PBM has a spatial resolution of $0.1942 \mu \mathrm{m}$ in the plane of the lens and $1 \mu \mathrm{m}$ along the propagation axis, in order to ensure algorithm convergence (Fig. 2a). As the optimum we have found a spacer with a length of $100 \mu \mathrm{m}$ and nanostructured GRIN lens with a diameter of $19.42 \mu \mathrm{m}$ and thickness of $40 \mu \mathrm{m}$. The GRIN lens with those dimensions and refractive indices should have a quarter pitch length of $67 \mu \mathrm{m}$. According to the simulation we expect a focus with a full width half maximum (FWHM) of $3.2 \mu \mathrm{m}$ at the working distance of $32 \mu \mathrm{m}$ (Fig. 2).

We have fabricated nanostructured GRIN lenses using the stack and draw method. All the procedure of nanostructured optical elements development has been described in detail in [6]. The process starts with the assembly of preforms of glass rods made of two different glasses. We use glass rods made of the two silicate glasses NC34 and NC21 [7]. The preform has been integrated and scaled down int a fiber drawing tower. A fiber $123 \mu \mathrm{m}$ in diameter with a nanostructured lens in the centre scaled down to $19 \mu \mathrm{m}$ at the diagonal has been achieved. The fiber is placed in a groove cut in a glass plate. The glass plate with the fiber attached is then cut with a diamond saw into $0.4 \mathrm{~mm}$ slices (Fig. 3). Next, the glass holders with a nanostructured lens are attached with mounting wax to a base plate and polished into the final lens samples with a thicknesses of $12 \mu \mathrm{m}$ using standard fiber polishing equipment.

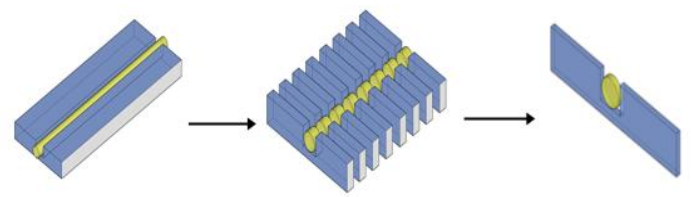

Fig. 3. Process of nanostructured GRIN lens development from the drawn fiber. A glass plate is achieved with a lens inside the groove.

Finally, the nanostructured GRIN lens and spacer were integrated with single mode optical fibers using active alignment and UV-curing optical adhesive for final fixation (Fig. 4). A microscope station with a manual alignment system was used. The alignment between the components with an accuracy of $2 \mu \mathrm{m}$ is achieved. A similar approach was recently used to integrate nanostructured GRIN axicon to a single mode fiber [8].

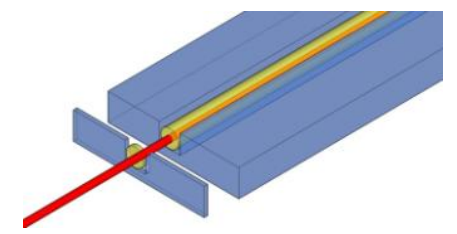

Fig. 4. Integration of a single mode fiber with a spacer and nanostructured GRIN lens. Active alignment and UV-curing optical adhesive was used. 
The optical setup integrated at the end of the fiber consists of a glass spacer with a thickness of $71 \mu \mathrm{m}$ and the lens a thickness of $12 \mu \mathrm{m}$ on top of it (Fig. 5). Taking into account the lens diameter and refractive index change the $12 \mu \mathrm{m}$ lens corresponds to 0.12 pitch length of the lens.

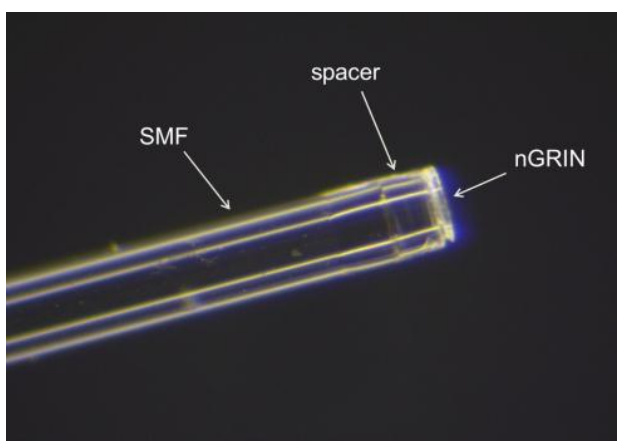

Fig. 5. Single mode fiber integrated with a nanostructured GRIN lens. A solid glass spacer allow aperture matching between the fiber core and aperture of the lens

The fiber terminated with a nanostructured GRIN microlens was characterized to verify its effective parameters. We used a $1550 \mathrm{~nm}$ laser coupled into a single mode fiber as a light source. Light after propagating through the lens was magnified by a $20 \times$ microscope objective $(\mathrm{NA}=0.35)$ and then projected onto a $\mathrm{CCD}$ camera operating with a fixed gain in the linear regime (Fig. 6). A phosphor coated analogue NIR CCD camera with a resolution of $720 \times 576$ pixels was used. The focal plane of the lens was determined by scanning the imaging system along the optical axis, with the translation resolution of such measurement being $\pm 3 \mu \mathrm{m}$ (Fig. 7).

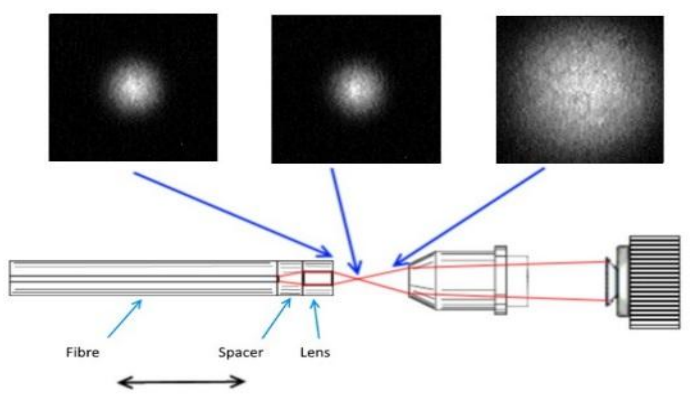

Fig. 6. Experimental setup for characterization of nanostructured GRIN microlenses.

We measured the focal plane at a working distance of $80 \mu \mathrm{m}$, which is larger than original simulation results. At that distance, the full width at half maximum (FWHM) of the focal spot is equal to $8 \mu \mathrm{m}$. The discrepancy between the modelling and experimental results is mainly related to differences between the sizes simulated and developed nanostructured lens. The simulated lens had a length of
$40 \mu \mathrm{m}$ corresponding to 0.15 pitch, while we have fabricated a lens with a length of $19 \mu \mathrm{m}$, which corresponded to 0.07 pitch. As a consequence, the working distance and FWHM at the focus obtained experimentally are larger than predicted.

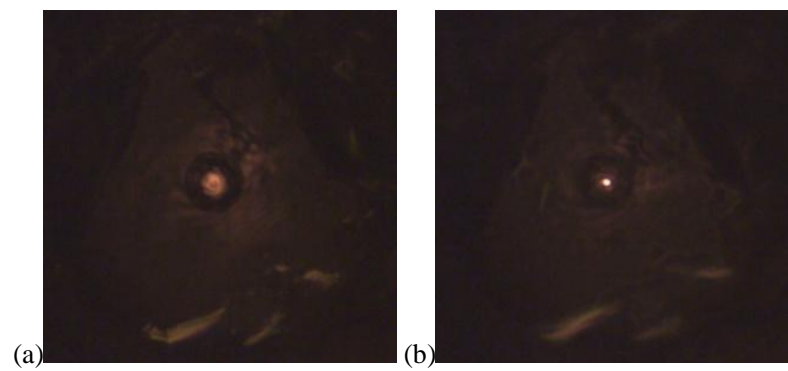

Fig. 6. Verification of lens performance: (a) intensity distribution at the lens facet, (b) intensity distribution at the distance of $80 \mu \mathrm{m}$ form the lens facet.

To conclude, we have reported the design and fabrication of a nanostructured GRIN microlens integrated with optical fibers. The fabricated lens has an effective parabolic profile of refraction. As measured, the lens produces a sharp focus with a FWHM beam diameter of $8 \mu \mathrm{m}$ at $80 \mu \mathrm{m}$ distance from the end facet of the lens. The fiber-integrated GRIN lens has an external diameter equal to that of the fiber. To our knowledge, this is the most compact fiber-GRIN lens system reported up to now. It can be applied in various optofluidic systems where the fiber with compact focusing functionality is required, offering the flexibility of integration with lab-on-chip systems. The working distance can be easily changed when GRIN lens thickness is modified.

This work was supported by the project National Science Centre in Poland (UMO-2013/10/M/ST3/00708) and COST Action MP1205 Advances in Optofluidics: Integration of Optical Control and Photonics with Microfluidics.

\section{References}

[1] M. Woerdemann, C. Alpmann, M. Esseling, C. Denz, Laser Photon. Rev. 7, 839 (2013).

[2] E. Weber, F. Keplinger, M.J. Vellekoop, IEEE Sensors J. 13, 4773 (2013).

[3] F. Hudelist, J.M. Nowosielski, R. Buczynski, A.J. Waddie, M.R. Taghizadeh, Opt. Lett. 35, 130 (2010)

[4] J.M. Nowosielski, R. Buczynski, F. Hudelist, A.J. Waddie, M.R. Taghizadeh, Opt. Comm. 2831938 (2010).

[5] A.Sihvola, Electromagnetic Mixing Formulas and Applications (London, The Institution of Electrical Engineers 1999).

[6] J. Nowosielski, R. Buczynski, A. J. Waddie, A. Filipkowski, D. Pysz, A. McCarthy, R. Stepien, M.R. Taghizadeh, Opt. Expr. 2011767 (2012).

[7] R. Stepien, J. Cimek, D. Pysz, I. Kujawa, M. Klimczak, R. Buczynski, Opt. Eng. 53, 071815 (2014).

[8] A. Filipkowski, B. Piechal, D. Pysz, R. Stepien, A. Waddie, M.R. Taghizadeh, R. Buczynski, Opt. Lett. 40, 5200 (2015). 